\title{
Características de la logística y la infraestructura de Puerto Antioquia en las exportaciones antioqueñas
}

\author{
Characteristics of logistics and infrastructure \\ Puerto Antioquia in Antioquia exports
}

Recibido: 19-05-2020 • Aprobado: 23-11-2020 • Página inicial: 169 - Página final: 193

\author{
María Isabel Guerrero Molina* \\ Yasmín Alexandra Vásquez Suárez**
}

\begin{abstract}
Resumen: la construcción de un puerto marítimo es una oportunidad de crecimiento para la región. El objetivo de la investigación buscó definir las características de la logística y la infraestructura de Puerto Antioquia en Urabá, y su competitividad frente a los demás puertos en Colombia. La metodología implementada en la investigación fue descriptiva, de corte cualitativa; la recopilación de la información se realizó a través de entrevistas semiestructuradas a 11 empresas, se eligieron dos empresas por cada sector que interviene en la operación logística: navieras, transportista terrestre, agencia de aduana, proveedores, tercero beneficiado de puertos y una comercializadora. Los hallazgos indican que los actores que intervienen en la operación logística están preparándose para realizar la operación automatizada, y en cooperación con las empresas de la región, realizando cambios y aplicando estrategias competitivas para mejorar la calidad de vida de la comunidad.
\end{abstract}

Palabras clave: comercio internacional; infraestructura de transporte; instalación portuaria; transporte marítimo.

\begin{abstract}
The maritime port construction is an opportunity to growth for the region, the objective of the research sought to define the characteristics of the logistics and infrastructure of Puerto Antioquia in Urabá and its competitiveness compared to other ports in Colombia. The methodology implemented in the research was descriptive of a qualitative nature, the collection of information was carried out through semi-structured interviews with 11 companies, two companies were chosen for each sector that intervenes in the logistics operation: shipping companies, land transporter, customs agency, suppliers, third party beneficiary of ports and a trading company. The findings indicate that the actors involved in the logistics operation are preparing to carry out the automated operation, and in cooperation with companies in the region made changes and applying competitive strategies to improve the quality of life of the community.
\end{abstract}

Keywords: International trade; transport infrastructure; port installation; maritime transport.

\footnotetext{
* Magíster en Negocios Internacionales, docente de tiempo completo e integrante del grupo de investigación Observatorio Público del Tecnológico de Antioquia, Colombia, integrante de la línea de investigación Comercio Exterior y Negociación del Grupo de investigación ECOSOL de la Facultad de Ciencias Administrativas, Económicas y Contables de la Universidad Católica Luis Amigó, Medellín-Colombia. maria.guerrerool@amigo.edu.co,

ORCID: https://orcid.org/0000-0002-8792-0832

** Magíster en Negocios Internacionales, docente tiempo completo e integrante de la línea investigación Comercio Exterior y Negociación del Grupo de investigación ECOSOL de la Facultad de Ciencias Administrativas, Económicas y Contables de la Universidad Católica Luis Amigó, Medellín-Colombia.

yasmin.vasquezsu@amigo.edu.co

ORCID: https://orcid.org/0000-0001-6926-5283
} 


\section{Características da logística e das infraestruturas Puerto Antioquia nas exportações antloqueñas}

Resumo: a construção de um porto marítimo é uma oportunidade de crescimento para a região. O objetivo da pesquisa buscou definir as características da logística e infraestrutura de Puerto Antioquia em Urabá, e sua competitividade em relação a outros portos da Colômbia. A metodologia implementada na pesquisa foi descritiva, qualitativa; A coleta de informações foi realizada por meio de entrevistas semiestruturadas com 11 empresas, sendo escolhidas duas empresas para cada setor que intervém na operação logística: armador, transportador terrestre, despacho aduaneiro, fornecedores, terceiro beneficiário dos portos e empresa comercial. Os resultados indicam que os atores envolvidos na operação logística estão se preparando para realizar a operação automatizada, e em cooperação com empresas da região, fazendo mudanças e aplicando estratégias competitivas para melhorar a qualidade de vida da comunidade. Conclui-se que Puerto Antioquia será a porta de entrada para o Caribe; trará desenvolvimento para a região do Urabá, proporcionando rotas comerciais, abrindo oportunidades e capacidade de empreendedorismo para a comunidade em geral; a construção de estradas e rodovias proporcionará os principais benefícios e esta região deve se preparar para o impacto positivo da economia nos diferentes setores: turismo, saúde e outros, que acompanham a expansão e gestão do comércio exterior.

Palavras-chave: comércio internacional; infraestrutura de transporte; instalação portuária; transporte marítimo. 


\section{Introducción}

Diferentes gobiernos, empresarios y productores han proyectado la región del golfo de Urabá como una zona de desarrollo y mejoramiento en la competitividad para el comercio exterior; Colombia les ha dado una proyección a los puertos ubicados en el Caribe: Cartagena, Barranquilla y Santa Marta, o en el Pacífico, con el puerto de Buenaventura; por su ubicación, posición estratégica y trayectoria en su funcionamiento.

Diferentes sectores económicos y gobernantes han visionado un puerto en la zona de Urabá que podría conllevar a más beneficios para el departamento de Antioquia y, en especial, para los urabaenses, generando empleo, mejorando los accesos viales y brindando un desarrollo a la región en diferentes mercados. En la investigación realizada, se plantea la identificación de las fortalezas de la logística e infraestructura para el proyecto Puerto Antioquia. En la región, existen las comercializadoras que han realizado los procesos de exportación e importación de manera eficiente y eficaz, su mercado principal ha estado orientado al transporte de productos de agricultura y comercialización de productos perecederos, como: el plátano, banano, aguacate, piña, gulupa, entre otros.

Sin embargo, estas comercializadoras han incursionado en el comercio de otro tipo de productos, tanto perecederos como no perecederos, lo que les ha permitido a diferentes empresarios poder realizar las operaciones de comercio exterior y acceder por las terminales de Uniban y Banacol (Zona Logística, 2019). Otros mercados relevantes de la zona están orientados hacia el corte y tala de madera, el ganado y la elaboración de artesanías en iraca (Alcaldía de Apartadó, 2019), estos también son comercializados a través de las comercializadoras, con el fin de ampliar los destinos de conexión con el exterior.

El proyecto Puerto Antioquia, se va a analizar desde la postura con la que cuenta las empresas que realizan los procesos de comercialización internacional, las cuales se encuentran ubicadas en el interior del país; esto es un hecho que le permite a la administración del departamento de Antioquia estar a la vanguardia de los proyectos, reformas y planeación de beneficios portuarios como el mejoramiento y construcción de carreteras, con el fin de disminuir las distancias entre los puntos de distribución; así mismo, mejorar el servicio de transporte hacia Urabá, y para que el proyecto pueda alcanzar éxito, se suman a otros proyectos como el mejoramiento de las carreteras y la infraestructura logística, los cuales han tenido avances significativos; y cuando las construcciones finalicen, tendrán un impacto positivo para las operaciones logísticas del departamento de Antioquia y en especial para la región de Urabá. 


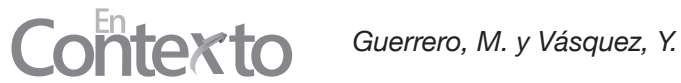

\section{Métodos y materiales}

El artículo plantea una investigación de tipo descriptivo y cualitativo de la realidad de las terminales comercializadoras que existen en Urabá para la exportación e importación de frutas y algunos productos determinados, con los cuales se llevan a cabo las operaciones portuarias. Posterior a ello, se realizó un análisis para establecer una representación de los aspectos más significativos a nivel logístico e infraestructural de los proyectos de Puerto Antioquia, Puerto Pisici y Puerto Darién, siendo el primero el foco de la investigación.

La recolección de la información se realizó por medio de entrevistas semiestructuradas, que ayudan a verificar la realidad de las terminales comercializadoras de Urabá, respecto al proyecto de Puerto de Antioquia y las demás concesiones aprobadas por el gobierno nacional. Se realizaron 11 entrevistas enfocadas a los gerentes, gerencia de servicios logísticos, gerencia de servicios sociales, directores de aduana, directores logísticos y ejecutivos comerciales; tres de estas empresas son socios del consorcio Puerto Antioquia. La cadena logística estudiada estuvo conformada por los transportistas terrestres, aduana, proveedores, terceros beneficiados de puertos, comercializadoras y navieras. Se realizó un rastreo de artículos científicos, material bibliográfico y bases de datos especializadas; igualmente, se consultó en páginas oficiales como la del Ministerio de Comercio, Industria y Turismo, Procolombia, Agencia Nacional de Infraestructura (ANI) y otras entidades que dan respuesta a las operaciones portuarias realizadas en el país.

\section{Resultados}

Se presentan los resultados teórico-conceptual de la investigación:

\section{Antecedentes}

Urabá es una subregión ubicada al norte al departamento de Antioquia, conformada por 11 municipios, con una extensión de $11.664 \mathrm{~km}^{2}$, considerada la "mejor esquina de Suramérica" (Aparicio, 2009), debido a que es el geocentro de Latinoamérica; cuenta con la única cordillera intertropical del mundo, la cual tiene una extensión de $450 \mathrm{~km}^{2}$, de los $1.500 \mathrm{~km}^{2}$ del Golfo de Urabá; está rodeada de aguas marítimas en el litoral Caribe (Gobernación de Antioquia, 2019).

Uno de sus municipios es Apartadó, se encuentra ubicado en la zona noroeste del departamento de Antioquia, hace parte del Urabá antioqueño; limita por el 
norte con el municipio de Turbo, por el oriente con el departamento de Córdoba, por el sur y occidente con el municipio de Carepa; la distancia a Medellín es de $344 \mathrm{Km}$. Apartadó cuenta con cuatro corregimientos, El Reposo, San José de Apartadó, Vijagual y Puerto Girón, además con 48 veredas, y es considerado como una zona con régimen aduanero especial, lo que significa la otorgación de estímulos a ciertas zonas del país que promueven el desarrollo industrial, comercial y turístico. El objetivo de esto es avanzar en el sector económico y fomentar empleo y la calidad de vida en la región correspondiente, logrando beneficios como facilidades aduaneras, no licencias de importación (Régimen Aduanero, 2018), etc. La distancia terrestre entre la capital de Antioquia, Medellín, y Apartadó es de $336 \mathrm{~km}^{2}$; el nombre de la ciudad, en el dialecto indígena, significa tierra de plátano.

El distrito de Turbo fue fundado en 1840, se encuentra a $32 \mathrm{~km}$ de Apartadó (por la carretera 62); tiene la mayor extensión de tierra del Golfo de Urabá, este territorio se considera líder en los cultivos de plátano, banano, maíz y yuca; además de ser un foco de desarrollo para los comerciantes e inversionistas (Banco de Desarrollo de América Latina, 2018).

Apartadó y Turbo son comunidades independientes, que comparten su desarrollo; su cercanía ha permitido que, en las últimas décadas, estén sujetos a la apertura de las carreteras desde el interior del país hacia el mar, con el fin de ser un territorio más industrializado. Según la Alcaldía del municipio de Apartadó, las actividades principales son la industria bananera, plátano, cacao, maíz, yuca, arroz, ñame, frutales, ganadería y tala de madera (Municipio de Apartadó, 2019), industria que se desarrolla en gran parte del territorio de Urabá.

Desde este marco territorial, en donde la biodiversidad y pluriculturalidad son una de las características más representativas del municipio y la región, hay un riesgo económico que puede llevar a una transformación social, debido a la presencia de las nuevas dinámicas industriales y comerciales que creará la operación portuaria debido a la falta de preparación de la comunidad para comprender las ventajas de Puerto Antioquia. Otro riesgo representativo será que puede generarse una economía excluyente hacia la población local, debido a la falta de preparación en temas puntuales que traiga consigo la construcción del puerto, actualmente sus recursos se derivan de los servicios eco sistémicos, los cuales se verían afectados por las operaciones portuarias (Echeverry et al., 2017).

La infraestructura portuaria en Urabá está compuesta por dos terminales privadas: Banacol y Uniban, estas empresas cuentan con instalaciones que atienden la necesidad de exportación de la industria de varias empresas locales y 


\section{Contex to Guerero,, , y visasues, $x$.}

nacionales; son comercializadoras internacionales y actúan como conglomerado industrial y comercial; en sus inicios realizaban la operación de exportación de banano y plátano, pero a medida que se consolidaron a nivel nacional, abrieron la comercialización a otras frutas, como piña, coco, aguacate, ñame, gulupa, entre otras, logrando especialización en el mercado de perecederos; y, a su vez, han abierto la operación de comercio exterior a otras empresas de otros sectores no perecederos, como el sector automotor, de cuidado y aseo personal, entre otros productos, con el fin de abrir nuevos mercados y oportunidades para la región.

El impacto cuando entre en operación Puerto Antioquia permitirá a la región de Urabá y al sector antioqueño promover el Golfo de Urabá y la franja del litoral en el cuadro del sistema portuario de Colombia para la exportación e importación de mercancías, bajo la potestad de pequeñas, medianas y grandes empresas con significativas dinámicas económicas, convirtiendo la región en un lugar de gran atractivo para la localización de nuevas empresas, trayendo consigo una alta competitividad en algunos términos importantes, como costos de transporte terrestre, menos distancia para la llegada de mercancías e infraestructura tecnológica, dinamizando la globalización del comercio internacional en términos de capacidad y competitividad, tanto a nivel local como nacional (Echeverry et al., 2017).

Se estima que la región de Urabá será el centro agroindustrial del país, a $300 \mathrm{~km}$ del Valle de Aburrá, y a menos distancia de los puertos actuales más cercanos, logrando planes complementarios para todo el sector social de la región, como la reactivación de proyectos educativos, orientados a la mano de obra calificada que se necesitará para la puesta en marcha de Puerto Antioquia, generando así nuevos empleos y agilidad para la comunidad en el traslado de sus productos. Se busca una contribución tributaria al desarrollo de las región desde el incremento de fuentes de empleo, dinamizados desde el sector de la construcción, restaurantes, hoteles y otros servicios, que tendrán oportunidades de expansión económica, gracias a la posibilidad del acercamiento del $95 \%$ de los buques del mundo, incluidos lo graneleros de 45.000 toneladas de capacidad de carga (Ecomining Desarrollo Sostenible, 2017).

\section{Terminales-Comercializadoras internacionales}

\section{Banacol}

Banacol nace en 1980 y hace parte de un grupo empresarial que lleva el mismo nombre, cuenta con 39 fincas propias bananeras que tienen alrededor de 7.000 hectáreas para la producción de banano; su principal destino de exportación de 
productos es el territorio europeo. Esta empresa es líder en el cultivo de banano, pero también produce piña de variedad Gold y comercializa con servicios logísticos; esta misma empresa tiene presencia en Costa Rica, y realiza la comercialización de productos con Estados Unidos, Canadá y Europa, gracias a las inversiones que la empresa ha realizado, ha ayudado a la comunidad en general disminuyendo los índices de desempleo.

Banacol es una operadora logística portuaria de Urabá. En el periodo de 2012 y 2014, la empresa redefine su modelo de negocios, gracias a la transformación logística que ha tenido la zona; anteriormente, se hacía la producción, comercialización y distribución integralmente de la fruta directamente en la terminal, se transportaba a la bodega (propia) en destino y se hacía la entrega al cliente final. Luego, se enfocaron en el esquema de comercialización, a la venta directa en el mercado por medio del término de negociación Free on board (FOB). Posterior a esto, en el año 2014, la empresa junto a su principal aliado, Dole Food Inc., que tiene el $90 \%$ de la producción, adquieren las fincas de banano de la empresa Banacol en Costa Rica (Comercializadora, comunicación personal, 18 de abril de 2019).

Toda la actividad productiva y logística de esta compañía se concentra en Urabá (Banacol, 2019).

La compañía implementó el sistema de identificación y comunicación GS1, el cual verifica el cumplimiento de los estándares globales para mejorar la cadena de abastecimiento (GS1 Colombia, 2019); cuenta con la certificación Customs Trade Partnership Against Terrorism (C-Tpat) de nivel III-Comercial contra el Terrorismo (FITAC, 2017), que son las mejores prácticas validadas y de inclusión que exceden los "Criterios de Seguridad Mínimos" (C-tpat, 2019).

En Urabá, la compañía cuenta con dos instalaciones, una de ellas se encuentra ubicada en Zungo y la otra en Nueva Colonia, en el municipio de Turbo; la operación se realiza por medio de barcazas, con una capacidad de transporte entre 4 y 12 contenedores, y equipos de carga entre 10 y 30 toneladas anualmente.

\section{Uniban}

C.I. Unión de Bananeros de Urabá S.A. (Uniban) inicia sus labores en 1966, con la razón social de empresa bananera Uniban, y en 1982 cambia para convertirse en comercializadora internacional. Su actividad comercial está concentrada en la región del Urabá antioqueño y tienen operaciones en la región del Magdalena y la Guajira (Uniban, 2019a). 


\section{Contex to Guerero,, , y rasasue,, .}

Desde 1975, la empresa Fyffes $N A$ es su principal aliada para comercializar los productos en la Unión Europea y en el Este de Europa. En el 2006, la empresa adquiere a la Promotora Bananera S.A. (Probán) en Santa Marta, esta negociación les permitió ganar posición comercial en Europa (Uniban, 2019b). La empresa cuenta con el Sistema de Gestión de Productividad (SGP), con el objetivo de buscar el incremento de la productividad, por medio de la aplicación de buenas prácticas agrícolas; también posee el Sistema de Información Geográfico (SIG), que permite monitorear los indicadores de productividad en los cultivos; cuenta con programas de formación en liderazgo, con el fin de impactar a los colaboradores y trabajadores; y para la tecnificación de los procesos tienen el Tropi Movil, que es una aplicación para registrar procesos de empaque, lectura de etiquetas y remisión de productos (Uniban, 2019c).

Estas dos terminales, tienen una trayectoria de más de 50 años, manejando operaciones de comercio exterior, si bien no hay puerto, realizan la operación portuaria, porque ya han consolidado una experticia, la cual será puesta en pro de la proyección de Puerto Antioquia.

\section{Líneas marítimas}

Las dos primeras líneas marítimas que entraron a operar en la zona de Urabá fueron CMA-CGM y HAMBURG-SUD, las cuales son las propietarias de las unidades de transporte marítimo: barcos o buques que operan las principales rutas marítimas mundiales. Este sector se caracteriza por la gran concentración de carga de importación y exportación que se maneja en el comercio internacional; las líneas navieras en la industria marítima contemplan todo tipo de mercancías como: general, granel, perecedera, peligrosa y frágil, siendo el medio de transporte más rentable en materia de fletes internacionales (Comercializadora, comunicación personal, 18 de abril de 2019). Según el Agente marítimo y representante portuario (Turbaduana, 2019), algunas de las líneas navieras que se manejan son:

- CMA-CGM Colombia S.A.S.

- Cool Carriers A.B. -Suecia.

- Dole Ocean Cargo Express -Costa Rica.

- Fyffes International -Costa Rica.

- GreenSea Chartering bvba -Bélgica.

- Hamburg Süd Colombia Ltda.

- Maersk Colombia S.A.

- Marfret Compagnie Maritime -Colombia. 
- Mediterranean Shipping Comprany -Colombia.

- SeaLand Colombia.

- Seaboard de Colombia S.A.

- Seatrade Reefer Chartering N.V-Bélgica.

- Star Reefers-UK.

- StreamLines N.V.-Curaçao.

Colombia es un país que tienen procesos de comercio exterior a través de los puertos en el Caribe y el Pacífico. Una vez estén en funcionamiento los puertos en el Golfo de Urabá, los cuales son Puerto Antioquia y Puerto Pisici, en Turbo; y Darién International Port, se proyectará al país como uno de los más competitivos en el ámbito internacional.

La producción de la región y de las zonas aledañas al departamento de Antioquia serán beneficiadas para la comercialización de sus productos, con precios competitivos, y la comercialización de los mismos en nuevos mercados. Desde la administración de Puerto Antioquia, en cabeza de sus socios comerciales, se prevé la sistematización de todos los procesos, a través de la mejora continua. No obstante, se debe establecer los limitantes antes de la construcción del puerto.

Según el entrevistado de la Aduana, (Aduana 1, comunicación personal, 17 de abril de 2019) los retrasos del inicio de la construcción del puerto se deben a circunstancias de temas legales, estos han retrasado el avance del proyecto; sin embargo, la concesión se encuentra vigente, existe el apoyo político y gubernamental y se están realizando encuentros con la comunidad.

Igualmente, para el entrevistado, una de las principales limitantes que tiene el inicio de la construcción del puerto es la posesión de tierras, los intermediarios cambiarios no desembolsaran dinero alguno, hasta que exista una resolución que indique que esos terrenos pertenecen a los reclamantes. Estos aspectos restrictivos hacen que la ejecución esté detenida y se denote incertidumbre entre los habitantes del sector de Turbo y Apartadó.

Los entrevistados de las dos comercializadoras indican que hoy en Urabá hay una operación portuaria, que, si bien no es un puerto, funciona como tal; las responsabilidades propias del comercio exterior se realizan y se hacen efectivas de acuerdo con la ley; la transformación logística que ha tenido la zona ha permitido tener avances significativos y eso está explicado en un cambio de modelo de negocios que ha operado en las empresas comercializadoras agrícolas, directamente desde la zona bananera (Comercializadora, comunicación personal, 18 de abril de 2019). 


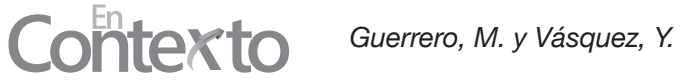

Para el entrevistado de la Aduana, (Aduana 2, comunicación personal, 17 de abril de 2019) la operación de Comercio Exterior en Urabá es pequeña, desde hace 20 años operan aproximadamente tres o cuatro agentes de aduana, con una zona aduanera pequeña en Turbo, los cuales recepcionan la carga de Panamá, en una operación donde no hay puerto, realizándose todo de manera rudimentaria, donde se hace descargue de la carga a granel y se traslada a diferentes depósitos aduaneros.

De acuerdo con una de las navieras (Naviera, comunicación personal, 16 de abril de 2019), países como Costa Rica se han consolidado como zonas especializadas en el manejo de la operación con contenedores refrigerados para exportación; por otra parte, Turbo tiene procesos similares a este tipo de operación, identificando dicha región con cambios positivos en la zona de Urabá, con el fin de mejorar los procesos de comercio exterior.

Es así como las compañías navieras están abiertas a transportar cualquier tipo de carga seca de otras industrias diferentes al banano, siempre y cuando cumplan con los requerimientos de seguridad marítima operativa para Uniban o Banacol; la percepción del mercado es el miedo o temor del cliente para iniciar cualquier operación por esta zona, por temas de seguridad y operatividad en Turbo. Esta es diferente a la que se maneja por el puerto de Cartagena o el de Buenaventura, y genera unos costos adicionales en el flete de exportación.

Ahora bien, en la práctica, el cliente final será quien realice la valoración de su preferencia respecto a los puertos y podrá tomar su decisión basada en costos, tiempo y procesos generados para realizar la operación. Puerto Antioquia presentará sus beneficios y oportunidades logísticas y de infraestructura frente a la operación de los otros puertos nacionales, los cuales tienen una trayectoria en comercio exterior.

Por otro lado, una de las agencias marítimas (Agenda Marítima, comunicación personal, 16 de abril de 2019), que cuenta con 45 años de experiencia, ha visto el crecimiento de la región, informando que el producto estrella ha sido la comercialización del banano $\mathrm{y}$, a su vez, ha visto la integración de otros productos perecederos y no perecederos, que se han venido potencializando a través de los años, como el aguacate, mango, tomates, flores, entre otros. Esta dinámica de comercialización de nuevos productos hace que las agencias marítimas se apoyen en las navieras para la creación de nuevas rutas de expansión, dinamizando los mercados compradores de Europa y Estados Unidos, los cuales son los representantes en mayor proporción dentro de la 
negociación para el transporte marítimo; mientras que las comercializadoras apoyan la labor logística dentro de la operación (montaje del barco como pago de faros, pago de armadores, consecución y pago de tripulación, corregir problemas de sanidad entre otros).

A su vez, los terceros beneficiados del puerto (Terceros Beneficiados del Puerto, comunicación personal, 19 de abril de 2019) y los transportistas terrestres señalan los frentes de la cadena logística, como lo son las fincas bananeras y otros productos agrícolas que se han ido potencializando en la zona, teniendo presente el método de siembra, riego, producción, empaque, embalaje, tramites logísticos y aduaneros, los cuales se dimensionan desde una perspectiva más ágil para la realización de cada uno de estos procesos (Transportistas Terrestres, comunicación personal, 19 de abril de 2019). Esto debido a la constante dinámica que saldrá de la construcción de los puertos, en relación a compras y ventas agrícolas; así mismo, el transporte terrestre se potencializará desde las vías Mar 1 y Mar 2, incluyendo el túnel del Toyo, lo que permitirá la reducción de los tiempos de tránsito al golfo de Urabá. Esta sinergia y relación de variables promoverán el desarrollo y sostenimiento de la región; en el futuro, las autopistas de la Prosperidad permitirán ahorros de tiempo y distancia, facilitando hasta un $25 \%$ de ahorro en los trayectos desde Medellín y el eje cafetero, y el 15 \% para Bogotá (Puerto Antioquia, 2019a).

Se realizaron entrevistas a dos de los proveedores que participan con la operación en las terminales comercializadoras en Turbo (Proveedor, comunicación personal, 20 de abril de 2019; Puerto Antioquia, 2019b), y estos concuerdan con el análisis que se hace a futuro del empleo en el Urabá antioqueño, con la proyección de la construcción de los tres puertos, la tendencia del empleo en Urabá se mira desde el aspecto demográfico: la transformación física territorial, los procesos de integración territorial y las apuestas productivas territoriales se enfocarán en el impacto de desarrollo de la región.

Con la construcción de estos puertos, la perspectiva crece desde una mirada de especialistas en diferentes áreas, tanto operativas como administrativas; de allí que se requieren personas con habilidades, capacidades y conocimientos en funciones con orientación definida hacia la parte de navieras, puertos, aduanas, logística del transporte marítimo, estibas, desestibas, agentes de carga, agentes de aduana, actualidad y normativa aduanera (Decreto 1165 del 2 de julio 2019), procedimiento operativo de importación y exportación, consecución de nuevos mercados, entre otros. 


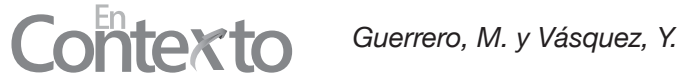

\section{Discusión}

\section{Puerto Antioquia}

El proyecto "Puerto Antioquia" nace en el año 2016, la Gobernación de Antioquia firma el 15 de noviembre de 2017 el acta de entendimiento para la construcción de Puerto Antioquia, con el fin de promover la construcción de un puerto internacional que permita impulsar los negocios para dinamizar la economía local y nacional (Uniban, 2017). El promotor y gestor del proyecto es PIO S.A.S., "[...] empresa holding dedicada a la implementación y gestión de servicios de infraestructura portuaria y logística" (PIO SAS, 2019).

Los socios del proyecto son CMA CGM, empresa naviera y de transporte de contenedores francesa dirigida por Jacques Saadé; es la tercera compañía mundial en el transporte de contenedores, operando 200 rutas marítimas entre 400 puertos en 150 países diferentes (CMA CMG, 2019). Para el entrevistado, desde la casa matriz en Marsella, la inversión en Puerto Antioquia es estratégica, un referente es la ubicación del puerto, el cual está directamente en el golfo de Urabá; el interés de CMA CGM de recalar en Turbo fue a raíz del potencial de exportación del banano que se maneja desde esta zona. Es así como esta compañía inicia la recalada con unos contratos globales que cerraron con algunas multinacionales de banano, las cuales son las grandes compradoras y comercializadoras a nivel mundial (Naviera, comunicación personal, 16 de abril de 2019).

Uniban, Banacol, Banafrut, CI Tropical, Agrícola Santamaría, Gobernación de Antioquia, Grupo Pío S.A.S. operarán con barcos de contenedores secos y refrigerados, vehículos, graneles secos, frutas y carga general (Uniban, 2017). El contrato de concesión se firmó entre la Sociedad Puerto Bahía Colombia de Urabá y la Agencia Nacional de Infraestructura (ANI) por 30 años; el terminal estará diseñado para buques portacontenedores de gran magnitud (Uniban, 2019d).

El proyecto contempla que el puerto sea multipropósito, con un calado de 15 metros, y un movimiento anual de 7 millones de toneladas de carga, capacidad de 1.200.000 TEU (contenedores normales de 20 pies) y tránsito de 90 mil vehículos. El puerto estará ubicado en el corregimiento de Nueva Colonia, en donde los socios tienen unos espacios de tierras que están conectados a la ubicación geográfica; allí se encuentran las zonas de fondeo y se proyecta la creación de un viaducto de $4.5 \mathrm{~km}$, para llevarlos desde tierra hasta altamar, ese viaducto es sobre el Río León y sobre la parte de fondeo, que está dentro 
del mar, es decir, en la misma zona (Aduanimex, comunicación personal, 09 de abril de 2019). Igualmente, la infraestructura que se proyecta está considerada de última generación, utilizando un sistema de identificación Optical Character Recognition (OCR), consiste en la identificación de manera óptima de mercancías para generar una fluidez en la entrada (Puerto Antioquia, 2019a).

La Sociedad Portuaria de Antioquia S.A.S. estará ubicada sobre el Atlántico, más cerca de los principales centros de producción y consumo del país. Además, está el Túnel del Toyo, una obra que tiene alrededor de 37.6 kilómetros de vía, que optimizará las actividades de comercio exterior de la región (PIO SAS, 2019). Estas obras tienen como meta la reducción de tiempos de tránsito entre Medellín y la región de Urabá, lo cual beneficiará la conectividad del sistema vial de otras zonas de país, incluyendo el centro del país.

Tabla 1

Distancia entre los municipios principales de Urabá y el resto del país

\begin{tabular}{cccccc}
\hline Ciudad & $\begin{array}{c}\text { Bogotá } \\
\left(\mathrm{km}^{2}\right)\end{array}$ & $\begin{array}{c}\text { Medellín } \\
\left(\mathrm{Km}^{2}\right)\end{array}$ & $\begin{array}{c}\text { Cali } \\
\left(\mathrm{Km}^{2}\right)\end{array}$ & $\begin{array}{c}\text { Manizales } \\
\left(\mathrm{Km}^{2}\right)\end{array}$ & $\begin{array}{c}\text { Armenia } \\
\left(\mathrm{Km}^{2}\right)\end{array}$ \\
\hline $\begin{array}{c}\text { Apartadó } \\
- \text { Turbo - } \\
\begin{array}{c}\text { Nueva } \\
\text { Colonia }\end{array}\end{array}$ & 739 & 340 & 779 & 534 & 607 \\
\hline Cartagena & 1101 & 643 & 1012 & 837 & 910 \\
\hline Barranquilla & 1095 & 708 & 1130 & 863 & 1082 \\
\hline Santa Marta & 970 & 858 & 1218 & 962 & 1051 \\
\hline
\end{tabular}

(Puerto Antioquia, 2019b).

La Autopista de la Prosperidad está diseñada bajo la concesión vial 4G, a este proyecto pertenece el Túnel del Toyo, que cuenta con 18 túneles, 29 puentes, los cuales atraviesan la cordillera occidental y cuenta con $16,7 \mathrm{~km}$ en vías nuevas, el túnel tendrá una extensión de $9,73 \mathrm{~km}$; el primer tramo es entre Cañas gordas y Giraldo y está a cargo de la Gobernación de Antioquia y la Alcaldía de Medellín (Figura 1) (Tunel del Toyo, 2019). 


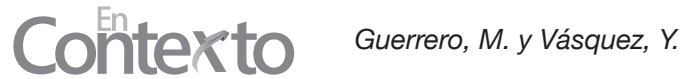

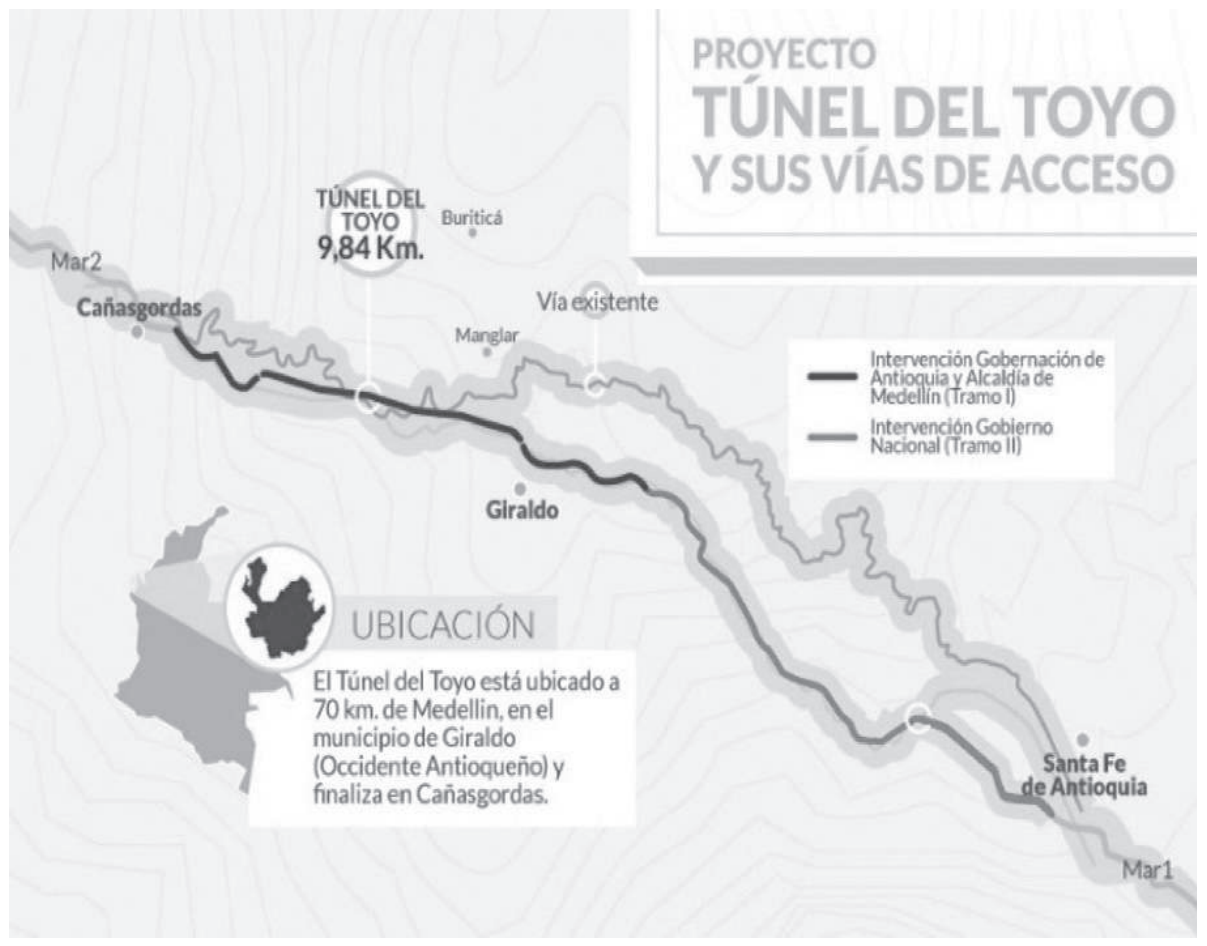

Figura 1. Proyecto Túnel del Toyo y sus vías de acceso.

Túnel del Toyo, 2019.

La conexión Mar 2 tiene una longitud de $250 \mathrm{~km}$, esta concesión se está ejecutando con el fin de mejorar la calzada actual en el tramo CañasgordasUramita, la operación y mantenimiento de la vía entre los municipios de Uramita y Dabeiba, permitieron un mejor trazado y la rehabilitación del resto del tramo entre Dabeiba-Mutatá-El Tigre y Necoclí.

Esta conexión vial tendrá 63 puentes, 15 túneles y 7 intersecciones viales, esta carretera no tendrá doble calzada (Autopistas Urabá, 2019). El proceso logístico para la llegada de contenedores a las terminales de Urabá se entiende bajo unos conceptos específicos que dan claridad en el proceso. De acuerdo con (DIMAR, 2019a), los siguientes términos se deben tener definidos para comprender la operación:

Fondeo: Anclar la embarcación dentro de aguas portuarias; Atraque: Colocar la embarcación en muelle o tierra, se detiene la carga, en este caso los contenedores; Desestiba: Proceso de manipulación de mercancía en el buque para bajarla y dejarla al costado de este; Estiba: Proceso de 
manipulación de mercancías con dos partes especificas entrada a la bodega y almacenamiento; Carga y descarga: Proceso en el cual la mercancía se encuentra en el medio de transporte terrestre (DIMAR, 2019a).

Durante este período, es preciso ejecutar una coordinación entre todos los involucrados que hacen parte de la operación de carga/descarga, autoridad del puerto, agente marítimo, estibadora, transportista, policía antinarcótico, ICA, DIAN y todos aquellos que se consideren necesarios, para planificar adecuadamente la operación de descarga y estar preparado con el apoyo logístico e infraestructura necesaria para la maniobra del buque, transporte terrestre y espacio suficiente en las terminales de recepción final del producto en el puerto.

La carga de banano o plátano es empacada en cajas de cartón en pallet y en container, traídas desde los embarcaderos de las distintas compañías bananeras, ubicadas en los canales de Zungo y Nueva Colonia, en bongos halados por remolcadores hasta la zona de cargue, frente a la desembocadura del Río León en tiempo de invierno, la cual queda a una distancia aproximada entre 4 y 7 millas náuticas, dependiendo del embarcadero; o a la zona de cargue frente al Río Leoncito en épocas de verano, la cual está a una distancia aproximada entre 8 y 13 millas náuticas, el mismo procedimiento se realiza para el descargue de insumos, que por el contrario son llevados a los distintos embarcaderos de las compañías bananeras.

Según el Centro de investigaciones oceanográficas e hidrográficas de Cartagena,

[1]os buques que realizan la ruta Turbo Coco Solo Turbo, estas embarcaciones por lo general son de bandera colombiana, una vez arriban al puerto atracan en el muelle de la estación de guardacostas a la espera de la visita oficial, de no presentar ninguna novedad posteriormente fondea frente a dicho muelle para empezar la maniobra de descargue, la cual se realiza a través de embarcaciones de madera con motores fuera de borda, las que se encargan de llevar las mercancías a tierra para ser distribuidas en las distintas bodegas. Abriendo las perspectivas en temas de tiempo, logística y costos a los usuarios de comercio exterior (DIMAR, 2019b).

La diferencia de la operación que se realiza en Urabá frente a los demás puertos radica en la maniobra, es decir, una motonave que atraca en la madrugada, se descarga en el menor tiempo posible programado, ese trayecto tiene una duración de alrededor de 12 horas; posterior a esto, entregan al agente de 


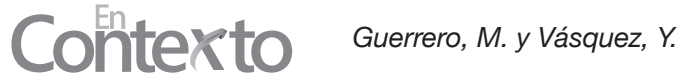

aduana, y llevan la mercancía para descargue en el muelle, esto quiere decir que, en tiempos óptimos, al día siguiente se tendrá un reflejo en el sistema aduanero de la DIAN.

De acuerdo con uno de los entrevistados, la región de Urabá es la aduana en Colombia, donde la operación aduanera de importación y exportación es más ágil y eficiente comparado con los demás puertos de Colombia, el poco volumen de carga hace que esta pueda ser planeada, en relación al proceso aduanero, cinco días antes de llegada la motonave y al arribar a la terminal sea entregada al agente de aduana, estando digitalizada para ser transmitida a la DIAN para pago de tributos aduaneros por medio de pago electrónico. Esto es debido a los pocos usuarios de comercio exterior que existen en el momento en esta zona, frente a un número bajo de operaciones realizadas, lo que permite tener un nivel de competitividad en los procesos operativos frente a los demás puertos (Aduana 1, comunicación personal, 17 de abril de 2019).

Puerto Antioquia busca una apertura frente a los procesos logísticos y operativos, esto incluye el ingreso y permanencia de líneas navieras y motonaves que admitan que el comercio exterior en Urabá fluya, las comercializadoras operan bajo dos terminales que pueden manejar aproximadamente cien operaciones mensuales; según esto, las exportaciones y las importaciones en Urabá no son muy frecuentes ni voluminosas, la nueva cara de esta región con carreteras avanzadas en 4G, obras de infraestructura comercial y vivienda hacen que el gremio industrial antioqueño, el eje cafetero y del interior del país coloquen su atención en esta zona, y Urabá se mire más por estrategia comercial (Aduanimex, comunicación personal, 09 de abril de 2019).

Para el entrevistado, la expectativa de las personas naturales y empresas jurídicas del sector del comercio exterior sobre la operación en pallets es considerada insegura y poco confiable técnicamente, pese a que esta logística en los últimos años ha sido ajustada, siendo cada vez más certera, según la experiencia y la trayectoria de las empresas agrícolas (Comercializadora, comunicación personal, 18 de abril de 2019). Con base en esta dinámica, para el año 2016 las líneas navieras dimensionaron su línea de negocio y comenzaron a llegar a la región de Urabá, no solo a almacenar banano, sino otro tipo de frutas como lo son: las fresas, uchuva, entre otros; es decir, diversificaron las cargas, y del año 2015 al 2016 se iniciaron los ajustes en los contratos agrarios; de esta manera, las grandes compañías compradoras de este tipo de productos abren su oferta de líneas marítimas diferentes a CMA CGM y HAMBURG-SUD, bajo unos requerimientos específicos que se ajustan a sus necesidades, entre las que comienzan a participar Maersk y MSC. 
Efecto de esto, es que en la actualizada la región de Urabá se ha dinamizado y se contempla con otra perspectiva de negocios a futuro; ahí están las grandes líneas marítimas del mundo, las cuales iniciaron una rotación y una concentración dedicada a la producción bananera. Empero, han realizado operaciones de comercio exterior con otro tipo de productos con conexiones a cualquier destino, abriendo acceso a otros mercados diferentes a los terrenos bananeros; para esto, los ajustes en las terminales se han ido incrementado por la diferencia de la operación bananera con las demás cargas, algunas de las particularidades que se han venido desarrollando son los cuartos fríos, de los cuales hay uno en Zungo y otro en Nueva Colonia, esto hace parte de una visión a futuro con desarrollo para la zona, aun antes de que se realice Puerto Antioquia.

Después de creado el puerto, las comercializadoras agrícolas deben escoger la terminal que las líneas marítimas de los clientes decidan; el negocio de Puerto Antioquia en Urabá es comercialización y venta de frutas, en donde se construyen alrededor de estos parques industriales que prestan todos los servicios logísticos operativos, enfocados a las cargas perecederas, para lo cual algunas de las empresas comercializadoras agrícolas han construido un conocimiento y aprendizaje integral de este tema, generando con esto confianza para los exportadores que no ven esta fortaleza en otros puertos (Santa Marta, Cartagena, Buenaventura o Barranquilla) (Banacol, 2019).

La consolidación de Puerto Antioquia, le apostará a la dinámica productiva de la región, proporcionando posibilidades de mayor expansión en el contexto internacional, gracias a oportunidades como la regulación del tráfico marítimo, planchones y remolcadores, que dejarán de transitar, permitiendo la recuperación del entorno natural del Río León y llevando, a su vez, un flujo de transporte de productos como banano, cacao, yuca, maíz, palma de aceite, ñame, arroz y piña, productos que han sido a lo largo del tiempo motor de la economía de la región. Según Ramírez Vásquez (2014), estas son algunas características de estos productos:

- El banano es el principal producto de comercialización nacional y exportación de la región del Urabá antioqueño. Se empezó a sembrar desde finales del siglo XIX, a través del consorcio colombo-alemán Albingia, seguido de la multinacional United Fruit Company-Chiquita, en 1960. El área bananera actual la componen aproximadamente 340 fincas que realizan sus exportaciones a través de: Banacol, Banafrut, Banur, coindex, Conserba, Tropical, Uniban. 


\section{Contexto Guerere, M. yvisower, $x$.}

- El cacao se ha convertido en uno de los productos más relevantes en la economía de la región, existe un gremio de productores en el Urabá antioqueño, quienes son en su gran mayoría, las personas que están sembrando y vendiendo cacao directamente a la Nacional de Chocolates (Compra el 65\% del cacao de la región) y Casa Lúker (Compra el 35\% de la cosecha); a su vez, una asociación de segundo piso (Monte Bravo), radicada en Turbo, pero que representa a once asociaciones del Chocó, está realizando ventas a mercados como Francia y Austria.

- La yuca es otro de los productos competitivos en la región, abasteciendo los mercados nacionales, destacándose los municipios de Mutatá, primer productor de la región, Arboletes, Turbo, Necoclí y San Pedro de Urabá, los cuales siguen como los grandes productores de la región. Urabá es el mayor productor de maíz en Antioquia; en el año 2014, se sembraron aproximadamente 16.245 hectáreas, con una producción de 25.845 toneladas para abastecer el mercado nacional.

- La palma de aceite es otro producto que ha tomado fuerza; hay sembradas aproximadamente 1.873 hectáreas (año 2014), las cuales fueron procesadas en la actual planta de extracción que se construyó en Guapá (Chigorodó). El Arroz es tradicional en la región de Urabá, se sembraron en el 2014, aproximadamente para autoconsumo, 7.546 hectáreas, con una producción de 11.138 toneladas; finalmente, el cultivo de Piña es considerado un cultivo promisorio, que permitirá diversificar la economía de Urabá.

- El ñame tiene relevancia en la agricultura de la región, siendo, al igual que los demás, un producto de alta competitividad en el mercado externo; aproximadamente para el año 2014, se sembraron en el norte de Urabá 820 hectáreas con una producción de 9.276 toneladas.

- La Región de Urabá es la mayor productora de ganado de Antioquia; sin embargo, el ciclo de bajos precios, así como la utilización de tierras ganaderas para otros negocios (reforestación y cultivos permanentes como palma de aceite), han hecho que este mercado haya perdido potencia dentro de la zona del Urabá (Ramírez Vásquez, 2014).

En la región de Urabá existen otros proyectos que van de la mano con el de Puerto Antioquia:

a) Puerto Turbo Pisici S.A., más conocido como Puerto Pisici, tiene como objeto la operación, mantenimiento y administración de puertos y terminales portuarias. El evento de la "Primera piedra" fue realizado el 12 de mayo de 2017. Estará ubicado en la cabecera urbana de la Bahía de Turbo, con una extensión de 39 hectáreas, las cuales lindan con caños Higinio, Casanova, 
las Yuquitas y el litoral de la bahía y en cercanías está ubicado el Aeropuerto Gonzalo Mejía, la DIAN y DIMAR (Puerto Pisici, 2019).

b) Darién International Port. La Sociedad Promotora de Infraestructura de Antioquia (Spiasa) suscribirá un contrato con una firma brasileña, que aportará los recursos para construir la primera fase del proyecto Darién International Port, ubicado en la zona rural de Necoclí, en Urabá (Construcción Latinoamericana, 2018). 
Contex to Guerrer, M. y vasaguze, $x$.

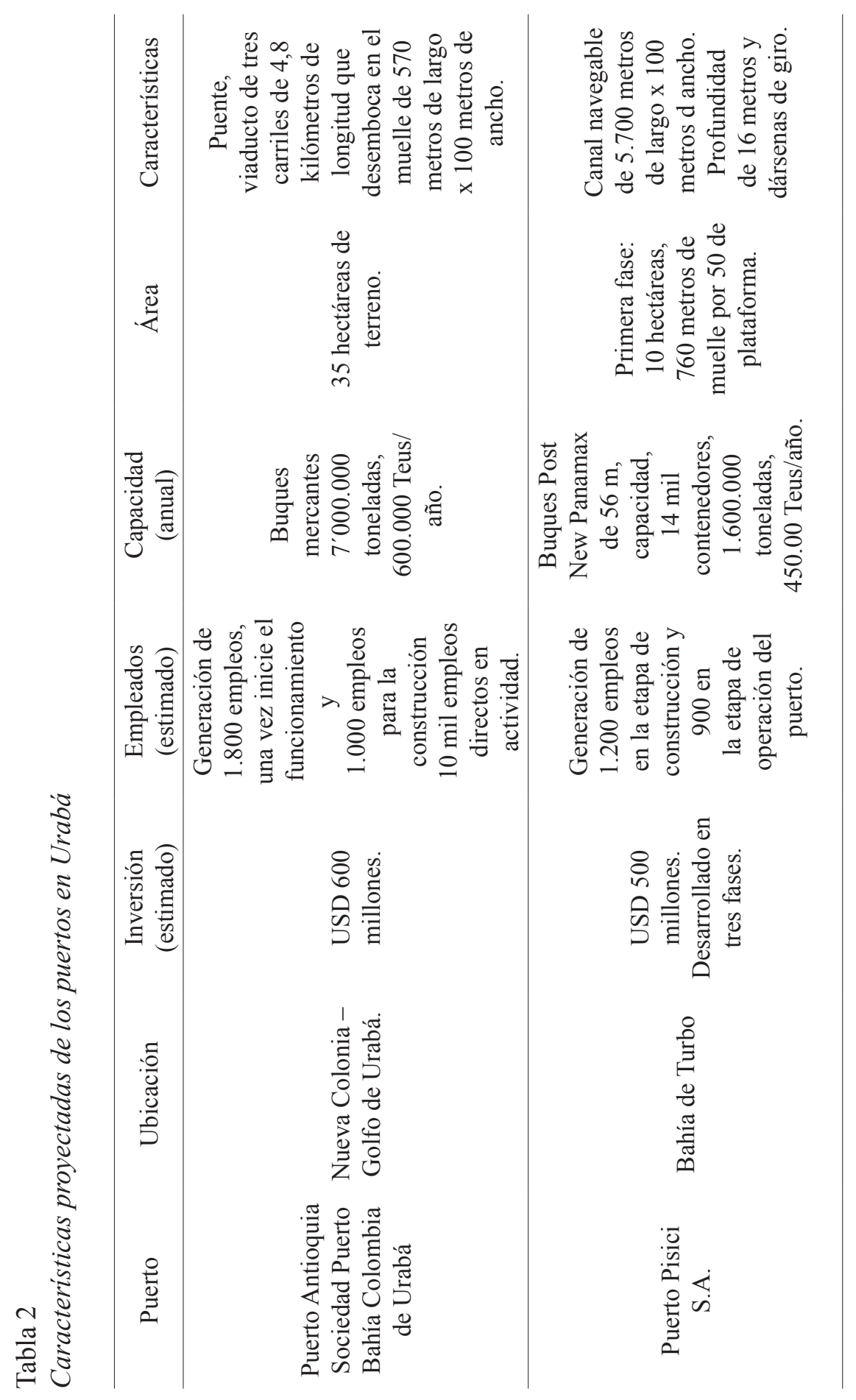


Características de la logística y la infraestructura...

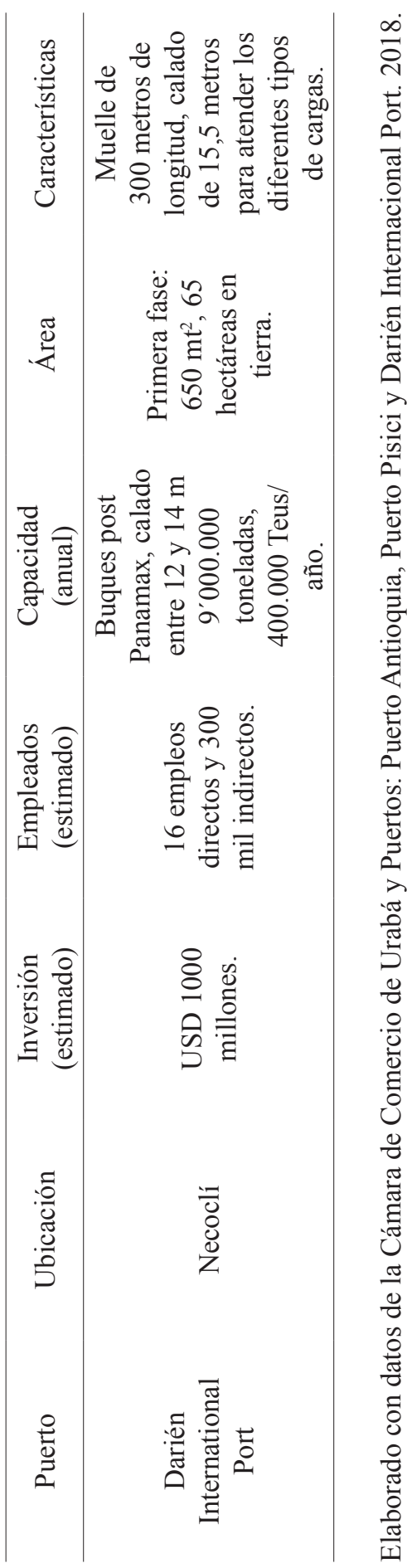




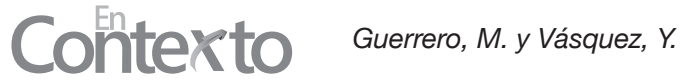

\section{Conclusiones}

Los empresarios colombianos se deben prepararse para ser aún más competitivos en el tema de comercio exterior; las acciones de mejoramiento que se están presentando en el país, en materia de logística (ampliación de las vías y proyectos de expansión portuaria), permiten que aquellos puedan realizar sus procesos de comercio exterior de manera asertiva.

Si bien el proyecto de Puerto Antioquia aún no inicia su construcción, por temas legales de adjudicación de tierras, una vez superado este tema, su apertura le permitirá a la zona tener un desarrollo no solo en los temas relacionados con los negocios internacionales, sino con la proyección de la zona como destino de negocios. Es ahí cuando el empresario colombiano debe concientizarse de la importancia de prepararse en materia tecnológica, investigativa e infraestructura de herramientas, ser más competitivo frente a la proyección de la región del Urabá antioqueño.

En la actualidad, los puertos del Caribe y el Pacífico son los pioneros en la gestión de las operaciones de exportación e importación, pero con el funcionamiento del puerto habrá una transformación en la nueva dinámica para la entrada y salida de productos en todos los puertos, atendiendo a aspectos como la reducción en distancias y costos en el transporte nacional. En la investigación se abordaron los puertos de Pisici y Darién, los cuales cuentan con las concesiones aprobadas. Una vez que Puerto Antioquia esté construido, se mejorarán las condiciones de competitividad para toda la cadena logística que interviene en el proceso de importación y exportación, como lo son las agencias de aduana, navieras, agencias de carga, proveedores, transportistas y todos los terceros involucrados dentro de la operación. Los proyectos de infraestructura vial, como Mar 1 y Mar 2, están en gestión; la inversión del gobierno departamental y nacional está enfocada en la influencia de la región, y se convertirá en una de las fortalezas de la zona del Urabá antioqueño. 


\section{Referencias}

Alcadía Municipal de Turbo. (28 de 04 de 2019). Alcadía Municipal de Turbo. ttp://www.turbo-antioquia.gov.co/municipio/nuestro-municipioA

Alcaldía de Apartadó. (11 de 11 de 2019). Mi Municipio. http://www.apartadoantioquia.gov.co/MiMunicipio/Paginas/Economia.aspx

Aparicio, J. (2009). La mejor esquina de surámerica: aproximaciones etnográficas a la protección de la vida en Urabá. Antípoda, 8, 87-115.

Autopistas Urabá. (19 de 06 de 2019). Autopistas Urabá. http://autopistasuraba.com/

Banacol. (14 de 06 de 2019). Banacol Historia. https://www.banacol.com/historia/

Banco de desarrollo deAmérica Latina. (2018). Una apuesta por la competitividad de Urabá. Instituto de Ciencia Política Hernán Echavarría Olózaga.

CMA CMG. (4 de 08 de 2019). https://www.cma-cgm.com/

Construcción Lanamericana. (12 de 02 de 2018). https://www.construccion-pa. $\mathrm{com} /$ noticias/proyecto-darien-international-port-sera-financiado-porfirma-brasilena/

C-tpat. (14 de 06 de 2019). Custumer Trade Partnership Against Terrorism. https://ctpatsecurity.com/level-3-spanish/

Decreto 1165. (02 de julio de 2019). Régimen de Aduanas. Número 51002.

DIMAR. (5 de 04 de 2019a). Centro de investigaciones oceanográficas e hidrográficas de Cartagena. https://www.cioh.org.co/derrotero/index. php?option $=$ com_content $\&$ view $=$ article $\&$ id $=188 \&$ Itemid $=229$

DIMAR. (5 de 11 de 2019b). Glosario DIMAR. https://www.dimar.mil.co/glosario

Echeverry, A., Velez, A., y Castaño, N. (2017). Entre el Agua y la Tierra Turbo, el reto del litoral. Una visión estratégica. Centro de Estudios Urbanos y Ambientales

Ecomining Desarrollo Sostenible. (2017). Análisis de gestión en investigación Puertos de Urabá. Ecomining. 


\section{Contex to Guerere,, . y vassuez, $x$.}

FITAC. (2017). XIX Congreso Internacional - Asamblea General de ASAPRA No. 48. Estudio solicitado por la Asociación Internacional de Operadores Económicos Autorizados Aduaneros y Logísticos-AIOEA y la Asociación Internacional de Agentes Profesionales de Aduanas - ASAPRA. SAPRA.

Gobernación de Antioquia. (13 de 06 de 2019). Gobernación de Antioquia. http://www.antioquia.gov.co/index.php/antioquia/regiones/uraba

GS1 Colombia. (05 de 04 de 2019). GS1 Colombia. https://www.gs1co.org/ nosotros/gs1global.aspx

Municipio de Apartadó. (14 de 05 de 2019). Municipio de Apartadó. http:// www.apartado-antioquia.gov.co/MiMunicipio/Paginas/Informacion-delMunicipio.aspx

PIO SAS. (04 de 06 de 2019). PIO SAS. http://www.puertoantioquia.com.co/ portal/es/acerca-de-pio-s-a-s.html

Presersa S.A. (2005). Procedimiento operativo normal para el manejo de granel seco.

Puerto Antioquia. (30 de 08 de 2019a). http://www.puertoantioquia.com.co/ portal/es/facilidades/infraestructura/zona-puerto-en-tierra.html

Puerto Antioquia. (27 de 09 de 2019b). http://www.puertoantioquia.com.co/ portal/es/ubicacion-puerto-antioquia.html

Puerto Pisici. (27 de 08 de 2019). https://puertopisisi.com/puerto-pisisi-una-realidad/

Ramírez Vásquez, E. (2014). Informe Socio Económico Camara de Comercio de Uraba.

Régimen Aduanero. (20 de 02 de 2018). Capitulo I - Zonas de régimen aduanero especial de Urabá, Tumaco y Guapi; Leticia; Inírida, Puerto Carreño, La Primavera y Cumaribo. Ministerio de Hacienda y crédito público.

Tunel del Toyo. (30 de 08 de 2019). https://tuneldeltoyo.com/proyecto/

Turbaduana. (30 de 09 de 2019). http://www.turbaduana.com/index.php/ alianzas/

Uniban. (15 de 11 de 2017). Uniban. http://uniban.com/index.php/es/categorianoticias/113-puerto-antioquia-mas-cerca-de-ser-realidad-en-uraba 
Uniban. (27 de 07 de 2019a). http://www.uniban.com/index.php/es/nosotros-2

Uniban. (13 de 06 de 2019b). Uniban. http://uniban.com/index.php/es/ nosotros-2/historia

Uniban. (27 de 09 de 2019c). http://www.uniban.com/index.php/es/nosotros-2/ calidad-y-productividad

Uniban. (20 de Marzo de 2019d). Uniban. http:/uniban.com/index.php/es/ categoria-noticias/174-se-firmo-contrato-de-concesion-de-puerto-antioquia

Zona Logística. (22 de 10 de 2019). Localización geoestratégica de Urabá. https://zonalogistica.com/costos-de-exportacion-e-importacion-atraves-de-uraba/

\section{Para citar este artículo:}

Guerrero, M. y Vásquez, Y. (2020). Características de la logística y la infraestructura de Puerto Antioquia, en las exportaciones antioqueñas. En-Contexto, 8(13), 169-193. 